\title{
24 TWO DATA ORGANIZATIONS FOR STORING SYMBOLIC IMAGES IN A RELATIONAL DATABASE SYSTEM
}

Aya Soffer and Hanan Samet

\author{
Computer Science Department and \\ Center for Automation Research and \\ Institute for Advanced Computer Science \\ University of Maryland at College Park \\ College Park, Maryland 20742 \\ aya@umiacs.umd.edu and hjs@umiacs.umd.edu
}

\begin{abstract}
A method is presented for integrating images into the framework of a conventional database management system (DBMS). It is applicable to a class of images termed symbolic images in which the set of objects that may appear are known a priori. The geometric shapes of the objects are relatively primitive and they convey symbolic information. Both the pattern recognition and indexing aspects of the problem are addressed. The emphasis is on extracting both contextual and spatial information from the raw images. A logical image representation that preserves this information is defined. Methods for storing and indexing logical images as tuples in a relation are presented. Indices are constructed for both the contextual and the spatial data, thereby enabling efficient retrieval of images based on contextual as well as spatial specifications. Two different data organizations (integrated and partitioned) for storing logical images in relational tables are proposed. They differ in the way that the logical images are stored. Sample queries and execution plans to respond to these queries are described for both organizations. Analytical cost analyses of these execution plans are given.
\end{abstract}

\subsection{INTRODUCTION}

Images (or pictures) serve as an integral part in many computer applications. Examples of such applications include CAD/CAM (computer aided design and manufacturing) software, document processing, medical imaging, GIS (geo- 
graphic information systems), computer vision systems, office automation systems, etc. All of these applications store various types of images and require some means of managing them. The field of image databases deals with this problem [8]. One of the major requirements of an image database system is the ability to retrieve images based on queries that describe the content of the required image(s), termed retrieval by content. An example query is "find all images containing camping sites within 3 miles of fishing sites".

In order to support retrieval by content, the images should be interpreted to some degree when they are inserted into the database. This process is referred to as converting an image from a physical representation to a logical representation. The logical representation may be a textual description of the image, a list of objects found in the image, a collection of features describing the objects in the image, a hierarchical description of the image, etc. It is desirable that the logical representation also preserve the spatial information inherent in the image (i.e., the spatial relation between the objects found in the image). We refer to the information regarding the objects found in an image as contextual information, and to the information regarding the spatial relation between these objects as spatial information. Both the logical and the physical representation of the image are usually stored in the database. An index mechanism based on the logical representation can then be used to retrieve images based on both contextual and spatial information in an efficient way.

There are many image database systems (e.g., Virage [18], QBIC [11], Photobook [13], FINDIT [17] as well as others $[2,5,6,12])$. Most systems treat the image as a whole, and index the images based mainly on color and texture. A few systems try to recognize individual objects in an image. These systems do not, however, address the issues of spatial relationship between the objects. Other systems deal with indexing tagged images (images in which the objects have already been recognized and associated with their semantic meaning) in order to support retrieval by image content.

In our work, we have chosen to focus on images where the set of objects that may appear are known a priori. In addition, the geometric shapes of these objects are relatively primitive and they convey symbolic information. Our application is the map domain where many graphical symbols are used to indicate the location of various sites such as hospitals, post offices, recreation areas, scenic areas etc. We call this class of images symbolic images. Other similar terms found in the literature are graphical documents, technical documents, and line drawings. Limiting ourselves to symbolic images simplifies object recognition enabling using well-known methods in document processing.

In this paper, we present methods for integrating symbolic images into a conventional database management system (DBMS). In our application, we make use of a relational DBMS although our ideas are applicable to other DBMS's. These methods offer solutions for both the pattern recognition and indexing aspects of the problem. We describe how to incorporate the results of these methods into an existing spatial database based on the relational model. 
Our emphasis is on extracting both contextual and spatial information from the raw images. The logical image representation that we define preserves this information. The logical images are stored as tuples in a relation. Indices are constructed on both the contextual and the spatial data, thus enabling efficient retrieval of images based on contextual as well as spatial specifications. It is our view that an image database must be able to process queries that have both contextual and spatial specifications, in addition to any traditional query.

We propose two different data organizations, termed integrated and partitioned, for storing images in relational tables. They differ in how logical images are stored. All of the examples and experiments in this paper are from the map domain. However, images from many other interesting applications fall into the category of symbolic images. These include CAD/CAM, engineering drawings, floor plans, and more.

The main contribution of this work lies in demonstrating how a traditional DBMS can be used to store and retrieve images and how partitioning this data effects the performance of the database. While the database and pattern recognition techniques that we use are well-known, the novelty of this work is in adapting and integrating these techniques into one system that provides a comprehensive solution for storing and retrieving images in a DBMS. We suggest solutions for all of the steps that are involved in this integration. These steps include: image acquisition, interpretation, storage, indexing, and retrieval. The main issues that need to be resolved are:

1. finding an image interpretation procedure whose results can be stored as entries in a traditional database in such a way that both the contextual and spatial information inherent in the image will be preserved.

2. what data organization is most suitable for the types of queries that are common in this application.

3. determining what strategies to use when computing answers to queries (i.e., how to use the double indexing on both contextual and spatial data efficiently).

4. finding ways to compute their costs.

The rest of this paper is organized as follows. We first present definitions as well as the notation used. Next, we outline the image input system used to convert images from their physical representation to their logical representation as they are input to the database. We continue by describing how images are stored in a database management system using the two data organizations that we propose including schema definitions and example relations. This is followed by sample queries along with execution plans and cost estimates for these plans. We conclude with some observations as well as directions for future research.

\subsection{DEFINITIONS AND NOTATIONS}

Below we define some terms and the notation used in the remainder of the paper. A general image is a two-dimensional array of picture elements (termed 
pixels) $p_{0}, p_{1}, \ldots, p_{n}$. A binary image is a general image where each pixel has one of two possible values (usually 0 and 1 ). One value is considered the foreground and the other the background. A general image is converted into a binary image by means of a threshold operation. A symbol is a group of connected pixels that together have some common semantic meaning. In a given application, symbols will be divided into valid symbols and invalid symbols. A valid symbol is a symbol whose semantic meaning is relevant in the given application. An invalid symbol is a symbol whose semantic meaning is irrelevant in the given application. A class is a group of symbols all of which have the same semantic meaning. All invalid symbols belong to a special class called the undefined class.

A symbolic image is a general image $I$ for which the following conditions hold: 1) Each foreground pixel $p_{i}$ in $I$ belongs to some symbol. 2) The set of possible classes $C_{1}, C_{2}, \ldots, C_{n}$ for the application is finite and is known a priori. 3) Each symbol belongs to some class. 4) There exists a function $f$ which when given a symbol $s$ and a class $C$ returns a value between 0 and 1 indicating the certainty that $s$ belongs to $C$.

Images can be represented in one of two ways. In the physical image representation, an image is represented by a two-dimensional array of pixel values. The physical representation of an image is denoted by $I_{\text {phys }}$. In the logical image representation, an image $I$ is represented by a list of tuples, one for each symbol $s \in I$. The tuples are of the form: $(C$, certainty,$(x, y))$ where $C \neq$ undefined, $(x, y)$ is the location of $s$ in $I$, and $0<$ certainty $\leq 1$ indicates the certainty that $s \in C$.

\subsection{IMAGE INPUT}

Conversion of input images from their physical to their logical representation is performed using methods common in document analysis [9]. These methods use various pattern recognition techniques that assign a physical object or an event to one of several pre-specified classes. Patterns are recognized based on some features or measurements made on the pattern. A library of features and their classifications, termed the training set library, is used to assign candidate classifications to an input pattern according to some distance metric. Each candidate classification is given a certainty value that approximates the certainty of the correctness of this classification.

We have adapted these methods to solve the problem of converting symbolic images from a physical to logical representation. Figure 24.1 is a block diagram of the image input system that we have developed for this purpose. It is driven by the symbolic information conveyed by the image. That is, rather than trying to interpret everything in the image, it looks for those symbols that are known to be of importance to the application. Any other symbol found in the image is labeled as belonging to the undefined class. This system is described in detail in [15]. In this paper we show how to integrate this system into a DBMS, thus we only give a short overview of the image input system here. A symbolic image $I_{\text {phys }}$ is input to the system in its physical representation. It is converted into 


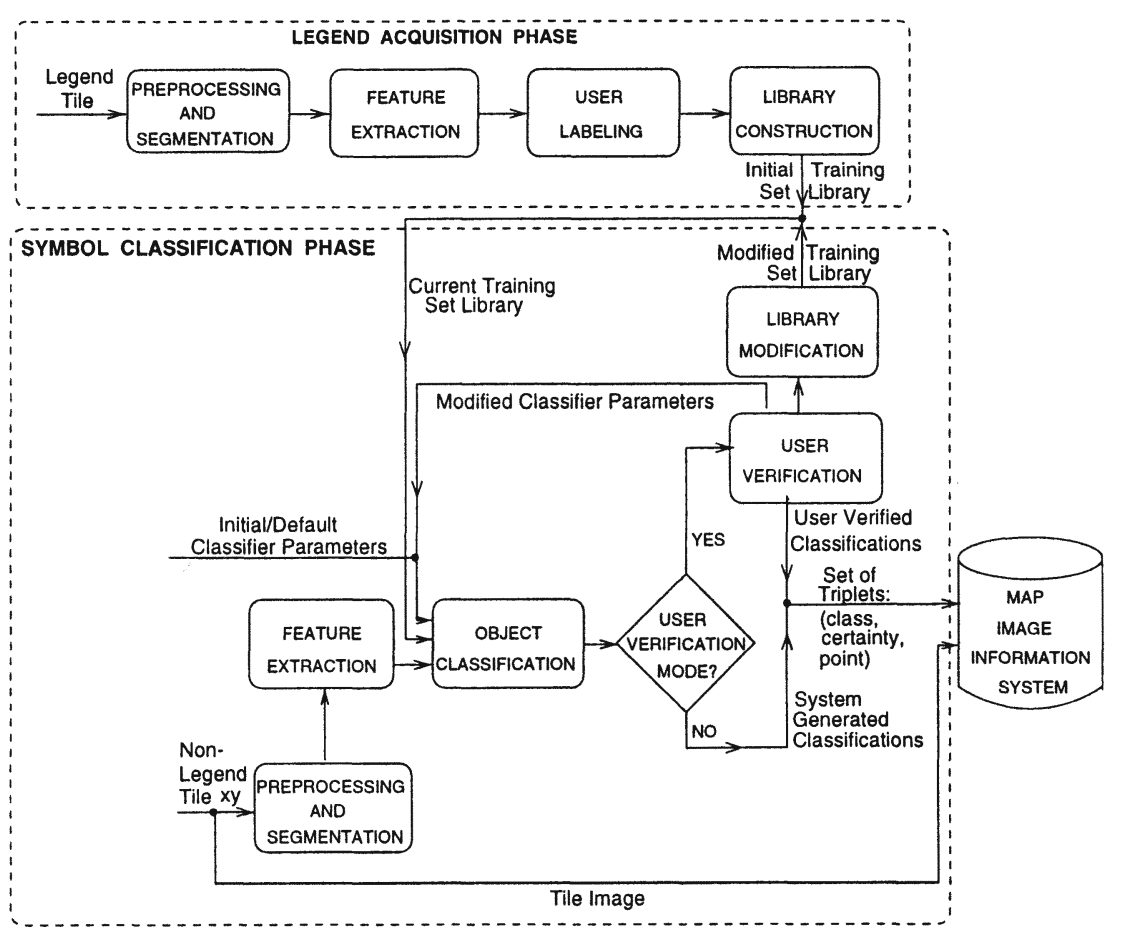

Figure 24.1: Image input system

a logical image by classifying each symbol $s$ found in $I_{\text {phys }}$ using the training set library. An initial training set library is constructed by giving the system one example symbol for each class that may be present in the application. In the map domain, the legend of the map may be used for this purpose.

The system may work in two modes. In user verification mode, users verify the classifications before being input to the database. The training set is modified to reflect the corrections that the user made for erroneous classifications. In automatic mode, classifications are generated by the system and input directly to the database. The user determines the mode in which the system operates. In general, the system should operate in user verification mode until the recognition rate achieved is deemed adequate. Then, the system can continue to process the input images automatically.

The output of applying the conversion process to $I_{\text {phys }}$ is a logical image where the tuples are of the form $(C$, certainty,$(x, y))$ where $C \neq$ undefined, $0<$ certainty $\leq 1$ indicating the certainty that $s \in C$, and $(x, y)$ is the location of $s$ in $I_{\text {phys }}$. For each image, a set of such tuples is inserted into a spatial database as described in the following section. In addition, the raw image $I_{\text {phys }}$ (i.e., the image in its physical representation) is also stored. 


\subsection{IMAGE STORAGE}

Images and other information pertaining to the application are stored in relational tables. The database system that we use for this purpose is SAND $[1,3]$ (denoting spatial and non-spatial database), developed at the University of Maryland. It is a home-grown extension to a relational database, in which the tuples may correspond to geometric entities such as points, lines, polygons, etc. having attributes which may be both of a locational (i.e., spatial) and a non-locational nature. Both types of attributes may be designated as indices of the relation. For indices built on locational attributes, SAND makes use of suitable spatial data structures. Attributes of type image are used to store physical images. Query processing and optimization is performed following the same guidelines of relational databases extended with a suitable cost model for accessing spatial indices and performing spatial operations.

We propose two different data organizations for storing the images in relational tables. They differ in the way logical images are stored. In the integrated organization, all tuples of the logical images are stored in one relation. In the partitioned organization, the tuples are partitioned into separate relations resulting in a one-to-one correspondence between relations and classes of the application. For example, tuples $(C$, certainty, $(x, y))$ of a logical image for which $C=C_{1}$ are stored in a relation corresponding to $C_{1}$. The motivation for the partitioned organization is that many queries in in an application using symbolic images need to access all symbols that are assigned the same classification. The part of the query that selects all tuples that belong to the same classification is repeated each time such a query is posed. The partitioned organization makes this repetitive selection at query time unnecessary by providing the option to partition the logical images relation. The partitioned organization is only suitable for applications in which the number of classes is relatively small, as there is one relation for each class and a proliferation of relations would make the database too complex. In the case of symbolic images, this is a reasonable assumption. The number of different symbols used to convey symbolic information (which corresponds to the number of classes) will most likely not be very large, otherwise it would be hard to keep track of or look up the semantic information that is conveyed by each symbol. For example, in the map domain this information must be contained in the legend of the map which is limited in space. Hence, the partitioned organization seems to be reasonable for a database that stores symbolic images. The partitioned organization also enables efficient use of spatial indices while processing spatial queries by using a spatial join operator (e.g., [14]).

\subsubsection{Integrated Organization}

The schema definitions given in Figure 24.2 define the relations in the integrated organization. We use an SQL-like syntax. The classes relation has one tuple for each possible class in the application. The name field stores the name of the class (e.g., star), the semant field stores the semantic meaning of 
(CREATE TABLE classes

name STRING PRIMARY KEY, semant STRING,

bitmap IMAGE);

(CREATE TABLE logical_images

img_id INTEGER REFERENCES physical_images(img_id),

class STRING REFERENCES classes (name),

certainty FLOAT (CHECK certainty BETWEEN 0 AND 1),

loc POINT,

PRIMARY KEY (img_id,class, loc));

Figure 24.2: Schemas for the relations classes, physical_images, and logical_images.

\begin{tabular}{|c|c|c|}
\hline class & semantics & bitmap \\
\hline $\mathrm{S}$ & harbor & (S) \\
\hline square & hotel & 0 \\
\hline scenic & scenic view & $\pi$ \\
\hline $\mathrm{T}$ & customs & (1) \\
\hline R & restaurant & (B) \\
\hline $\mathbf{P}$ & post office & (P) \\
\hline M & museum & (1) \\
\hline K & cafe & $\mathbb{B}$ \\
\hline waves & beach & $\theta$ \\
\hline triangle & camping site & $\theta$ \\
\hline B & filling station & (B) \\
\hline arrow & holiday camp & c \\
\hline cross & first aid station & 0 \\
\hline fish & fishing site & $\infty$ \\
\hline $\mathrm{H}$ & service station & $\Theta$ \\
\hline $\inf$ & tourist information & $\mathbf{0}$ \\
\hline pi & picnic site & (ii) \\
\hline air & airfield & (A) \\
\hline star & site of interest & * \\
\hline telephone & public telephone & (2) \\
\hline box & youth hostel & a \\
\hline U & sports institution & (1) \\
\hline
\end{tabular}

Figure 24.3: Example instance for classes relation.

the class in this application (e.g., site of interest). The bitmap field stores a bitmap of an instance of a symbol representing this class. It is an attribute of type IMAGE. The classes relation is populated using the same data that is used to create the initial training set for the image input system (i.e., one 


\begin{tabular}{|c|c|c|c|}
\hline image_id & descriptor & raw & upper_left \\
\hline image_1 & tile 003.012 of Finnish road map & Fig. 24.5 & $(6144,1536)$ \\
image_2 & tile 003.013 of Finnish road map & Fig. 24.6 & $(6656,1536)$ \\
\hline
\end{tabular}

Figure 24.4: Example instance for physical_images relation.

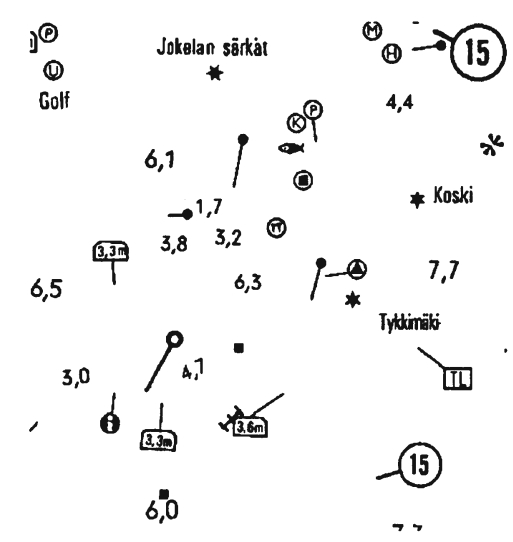

Figure 24.5: Example: image_1.

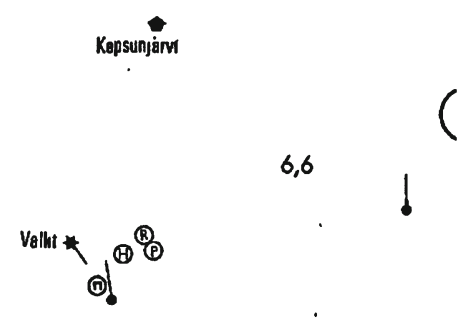

18,4

Figure 24.6: Example: image 2 .

example symbol for each class that may be present in the application along with its name and semantic meaning). See Figure 24.3 for an example instance of the classes relation in the map domain.

The physical_images relation has one tuple per image $I$ in the database. The img-id field is an integer identifier given to the image $I$ when it is inserted into the database. The descriptor field stores an alphanumeric description of the image $I$ that the user gives when inserting $I$ (this is meta-data). The raw field stores the actual image $I$ in its physical representation. It is an attribute of type IMAGE. The upper left field stores an offset value that locates the upper left corner of image $I$ with respect to the upper left corner of some larger image $J$. This is useful when a large image $J$ is tiled, as in our example map domain. Subtracting this offset value from the absolute location of $s$ in the the non-tiled image $J$ yields the location of $s$ in the tile $I$ that contains it. It is an attribute of type POINT. Any additional meta-data that the user may wish to store about the images such as how they were formed, camera angles, scale, etc. can be added as fields of this relation. See Figure 24.4 for an example instance of the physical_images relation in the map domain.

The logical_images relation stores the logical representation of the images. It has one tuple for each candidate class output by the image input system for each valid symbol $s$ in each image $I$. The tuple has four fields. The img_id field is the integer identifier given to $I$ when it was inserted into the database. 


\begin{tabular}{|c|c|c|c|}
\hline image_id & class & certainty & location \\
\hline image_1 & $\mathrm{M}$ & 1 & $(6493,1544)$ \\
image_1 & $\mathrm{P}$ & 0.99 & $(6161,1546)$ \\
image_1 & $\mathrm{H}$ & 0.99 & $(6513,1566)$ \\
image_1 & $\mathrm{U}$ & 1 & $(6167,1583)$ \\
image_1 & star & 0.99 & $(6332,1586)$ \\
image_1 & $\mathrm{P}$ & 0.99 & $(6432,1622)$ \\
image_1 & $\mathrm{K}$ & 1 & $(6416,1636)$ \\
image_1 & fish & 1 & $(6411,1661)$ \\
image_1 & scenic & 0.99 & $(6630,1662)$ \\
image_1 & square & 1 & $(6422,1693)$ \\
image_1 & star & 0.99 & $(6540,1712)$ \\
image_1 & pi & 0.99 & $(6396,1741)$ \\
image_1 & triangle & 1 & $(6475,1784)$ \\
image_1 & star & 1 & $(6474,1814)$ \\
image_1 & cross & 0.79 & $(6291,1854)$ \\
image_1 & box & 0.74 & $(6357,1862)$ \\
image_1 & inf & 1 & $(6226,1937)$ \\
image_1 & box & 1 & $(6280,2011)$ \\
image_2 & arrow & 0.99 & $(6861,1544)$ \\
image_2 & scenic & 0.72 & $(6803,1565)$ \\
image_2 & pi & 0.99 & $(6849,1756)$ \\
image_2 & $\mathrm{R}$ & 0.71 & $(6849,1756)$ \\
image_2 & $\mathrm{P}$ & 0.99 & $(6858,1771)$ \\
image_2 & $\mathrm{H}$ & 0.99 & $(6827,1775)$ \\
image_2 & $\mathrm{U}$ & 0.79 & $(6827,1775)$ \\
image_2 & $\mathrm{pi}$ & 0.99 & $(6800,1807)$ \\
image_2 & $\mathrm{R}$ & 0.99 & $(6800,1807)$ \\
\hline
\end{tabular}

Figure 24.7: Example instance for the logical_images relation in the map domain. The tuples correspond to the symbols in the images of Figures 24.5 and 24.6.

It is a foreign key referencing the img_id field of the tuple representing $I$ in the physical_images relation. The class and certainty fields store the name of the class $C$ to which the image input system classified $s$ and the certainty that $s \in C$. The loc field stores the $(x, y)$ coordinate values of the center of gravity of $s$ relative to the non-tiled image. See Figure 24.7 for an example instance of the logical_images relation in the map domain for the images given in Figures 24.5 and 24.6.

Constructing Indices Indices are defined on the schemas defined above as follows (in SQL-like notation): 


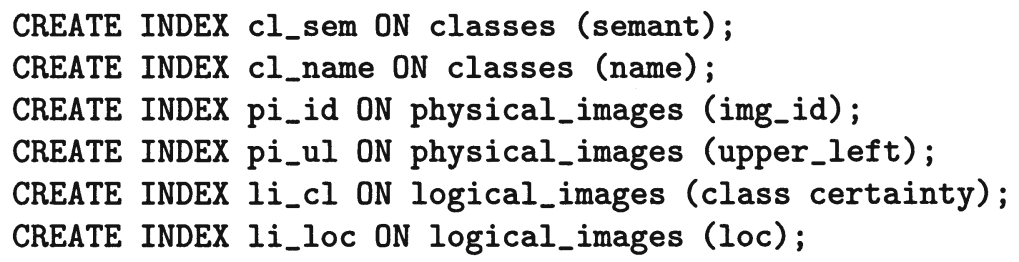

cl_sem and cl_name are alphanumeric indices. They are used to search the classes relation by semant and name, respectively. The pi_id index is also alphanumeric. It is used to search the physical_images relation by img_id. pi_ul is a spatial index on points. It is used to search the physical_images relation by the coordinates of the uppe. left corner of the images. li_cl is an alphanumeric index. It is used to search the logical_images relation by class. It has a secondary index on attribute certainty. Thus, tuples that have the same class name are ordered by certainty value within this index. li_loc is a spatial index on points. It is used to search the logical_images relation by location (i.e., to deal with spatial queries regarding the locations of the symbols in the images such as distance and range queries). The spatial indices are implemented using a PMR quadtree for points [10].

Observe that the file structures resulting from the integrated organization are very similar to the file structures used by inverted file methods for storing text [4]. An inverted file consists of two structures. A vocabulary list which is a sorted list of words found in the documents, and a posting file indicating for each word the list of documents that contain it and information regarding its position in the document. The vocabulary list is actually an index on the posting file, and is used to locate the record of the posting file corresponding to a given word on disk. In our organization, the logical_images relation corresponds to the posting file. The index li_cl on this relation plays the role of the vocabulary list. The main difference from text is that as we are dealing with 2-dimensional information rather than 1-dimensional information, we need more elaborate methods to store and index the locational information. In particular, just storing the location, as is done for text data, is insufficient. In order to answer spatial queries efficiently, these locations must be sorted by use of a spatial index. Figure 24.8 illustrates the file structures used following the integrated organization that correspond to similar file structures used for text data.

\subsubsection{Partitioned Organization}

In the partitioned organization, tuples are partitioned into separate relations resulting in a one-to-one correspondence between relations and classes of the application. For example, tuples $(C$, certainty,$(x, y))$ of a logical image for which $C=C_{1}$ are stored in a relation corresponding to $C_{1}$. Figure 24.9 gives schema definitions for relations of the partitioned organization corresponding to the logical_images relation of the integrated organization. Both the classes and physical_images definitions are identical to those in the integrated or- 


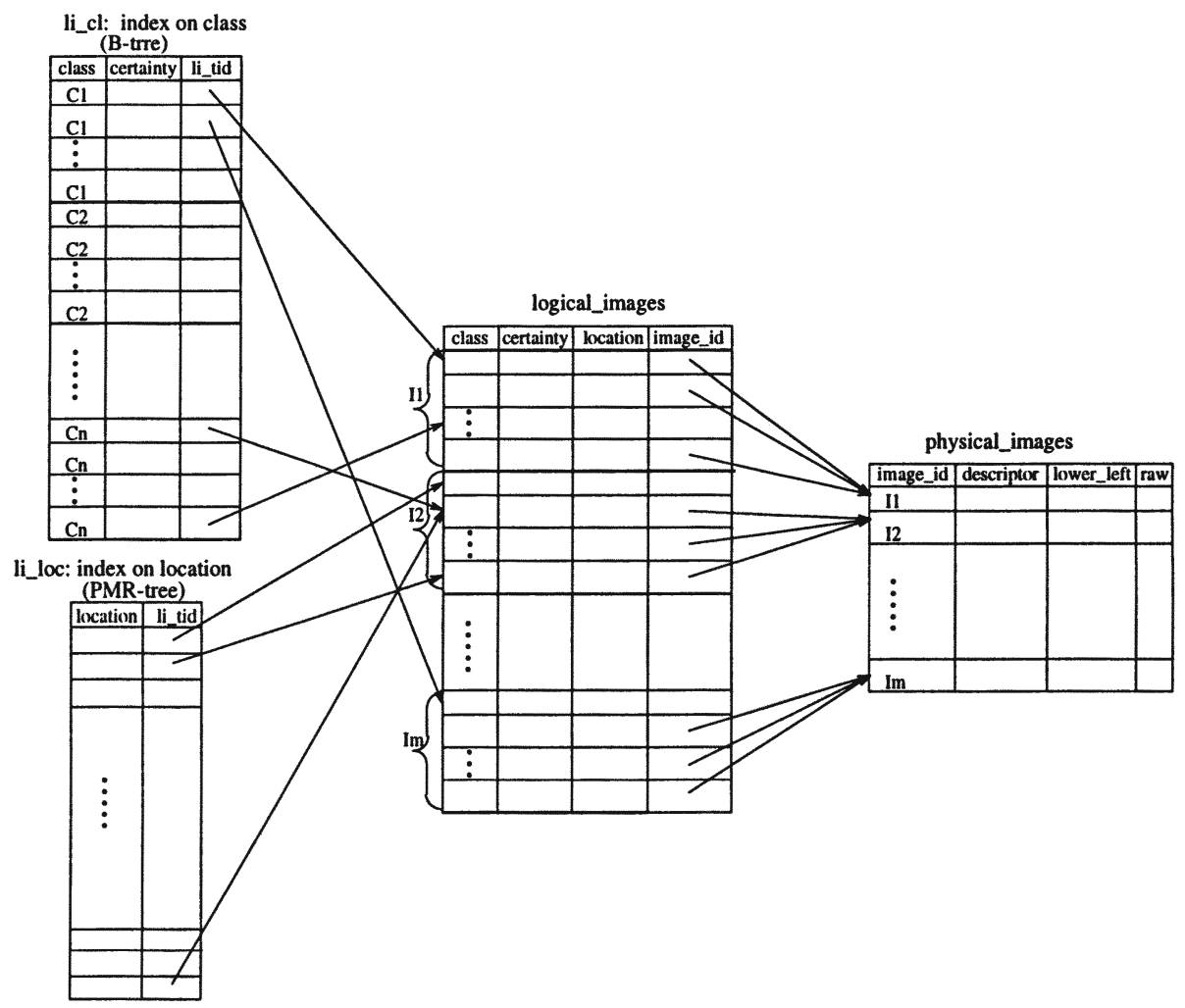

Figure 24.8: File structures for logical and physical images using the integrated organization.

ganization. The only difference between the organizations is the way the logical images are stored. In the partitioned organization, there is one relation, cl_part for each class $c l$ in the application. Each relation cl_part contains the logical images tuples $(C$, certainty,$(x, y))$ for which $C=c l$. This is equivalent to the result of a selection operation: SELECT FROM logical_images WHERE class $=c l$. See Figure 24.10 for example instances of relations star_part, P_part, scenic_part, and pi_part for the images in Figures 24.5 and 24.6.

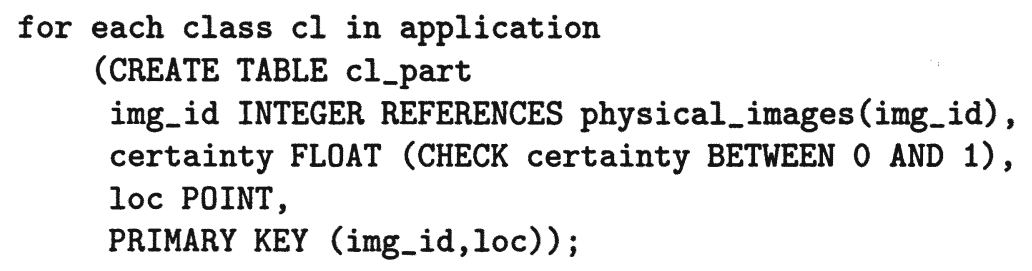

Figure 24.9: Schemas for the cl_part relations in the partitioned organization. 
star_part:

\begin{tabular}{|c|c|c|}
\hline image_id & certainty & location \\
\hline image_1 & 0.99 & $(6332,1586)$ \\
image_1 & 0.99 & $(6540,1712)$ \\
image_1 & 1 & $(6474,1814)$ \\
\hline
\end{tabular}

scenic_part:

\begin{tabular}{|c|c|c|}
\hline image_id & certainty & location \\
\hline image_1 & 0.99 & $(6630,1662)$ \\
image_2 & 0.72 & $(6803,1565)$ \\
\hline
\end{tabular}

P_part :

\begin{tabular}{|c|c|c|}
\hline image_id & certainty & location \\
\hline image_1 & 0.99 & $(6161,1546)$ \\
image_1 & 0.99 & $(6432,1622)$ \\
image_2 & 0.99 & $(6858,1771)$ \\
\hline
\end{tabular}

pi_part:

\begin{tabular}{|c|c|c|}
\hline image_id & certainty & location \\
\hline image_1 & 0.99 & $(6395,1741)$ \\
image_2 & 0.99 & $(6849,1756)$ \\
image_2 & 0.99 & $(6800,1807)$ \\
\hline
\end{tabular}

Figure 24.10: Example instances of relations star_part, scenic_part, P_part, and pi_part. The tuples correspond to the symbols in the images of Figures 24.5 and 24.6.

Constructing Indices Indices are defined on the separate class schemas of the partitioned organization as follows (in SQL-like notation):

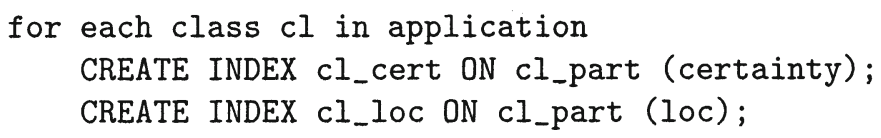

Each instance of the cl_part relation has an alphanumeric index on certainty and a spatial index on loc. The spatial index is used to deal with queries of the type "find all images with sites of interest within 10 miles of a picnic area" by means of a spatial join operator. Figure 24.11 illustrates the file structures for the partitioned organization corresponding to file structures used for text data. 


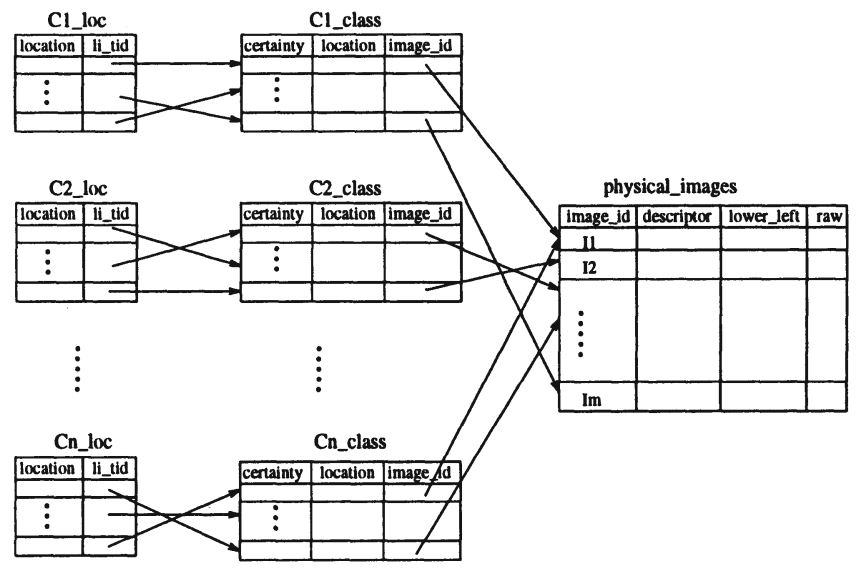

Figure 24.11: File structures for logical and physical mages using the partitioned organization.

\subsection{RETRIEVING IMAGES BY CONTENT}

As mentioned above, we distinguish between contextual information and spatial information found in images. Similarly, we distinguish between query specifications that are purely contextual and those that also contain spatial conditions. A contextual specification defines the images to be retrieved in terms of their contextual information (i.e., the objects found in the image). For example, suppose we want to find all images that contain fishing sites or campgrounds. A spatial specification further constrains the required images by adding conditions regarding spatial information (i.e., the spatial relations between the objects).

In order to describe the methods that we use for retrieving images by content, we first present some example queries. Next, we demonstrate the strategies used to process these queries. We conclude by analyzing the expected costs of these strategies (termed plans) and compare the data organizations (i.e., integrated and partitioned).

\subsubsection{Example Queries}

The example queries in this section are first specified using natural language. This is followed by two equivalent SQL-like queries. The first assumes an integrated organization and the second assumes a partitioned organization.

Query Q1: display all images containing a scenic view .

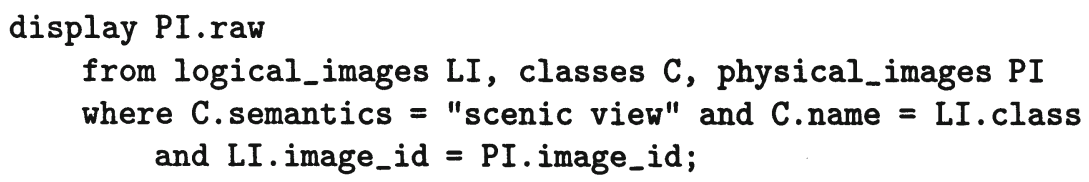


from scenic_part SC, physical_images PI

where SC.image_id = PI. image_id;

Notice that in order to write SQL-like queries for the partitioned organization, the names of the relations corresponding to each partition must be known. This can easily be overcome by having the system assign names to these relations. These names are derived from the class attribute of relation classes. Two functions that perform this name conversion are provided. get_rel_name returns the name of a relation given the class name. get_class returns the class name given a relation name. Thus, there is no need for the user to know the names assigned by the system to these relations.

Query Q2: display all images containing a scenic view within 5 miles of a picnic site.

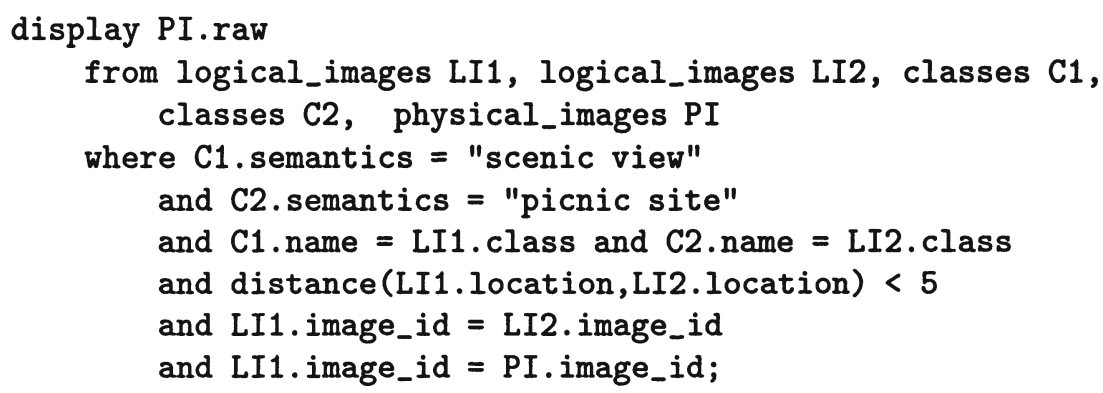

The function distance takes two geometric objects (e.g., two points) and returns a floating point number representing the Euclidean distance between them.

\subsubsection{Query Processing}

The following plans outline how responses to queries Q1 and Q2 are computed using the two data organizations. These plans utilize the indexing structures available for each organization. Indices on alphanumeric attributes are capable of locating the closest value greater than or equal to a given string or number. Indices on spatial attributes are capable of returning the items in increasing order of their distance from a given point (this is termed an incremental nearest neighbor operation) [7]. This operation may optionally receive a maximum distance, $D$, and it will stop when the distance to the next nearest neighbor is greater than $D$. Thus, it returns all neighbors within $D$ of a query point in increasing distance. Direct addressing of a tuple within a relation is possible 
by means of a tuple identifier (or tid for short). All index structures have an implicit attribute that stores this tid. The $X^{\text {th }}$ plan, labeled $\mathrm{P} x_{I}$, uses the integrated organization. The $X^{\text {th }}$ plan, labeled $\mathrm{P} x_{P}$, uses the partitioned organization.

Query Q1: display all images containing a scenic view.

Plan $\mathbf{P} 1_{I}$ : Search using an alphanumeric index on class.

Get all tuples of logicalimages which correspond to "scenic view"

(use index li_cl)

For each such tuple t

display the physical image corresponding to $t$

Plan $\mathbf{P} 1_{P}$ Search the scenic view partition sequentially

For each tuple t of the "scenic view" partition display the physical image corresponding to $\mathbf{t}$

Query Q2: display all images containing a scenic view within 5 miles of a picnic site.

Finding a suitable plan for query Q2 gives rise to many query optimization issues. Most of these issues are also applicable to spatial databases (e.g., [1]). To see the complexity of these issues, we give two different plans for computing an answer to query Q2 using each organization. The first uses only alphanumeric indices, while the second uses an alphanumeric index and a spatial index.

Plan P2A $\mathbf{A}_{I}$ Search picnic tuples and scenic view tuples using the alphanumeric index on class. For each picnic tuple, check all scenic view tuples to determine which ones are within the specified distance.

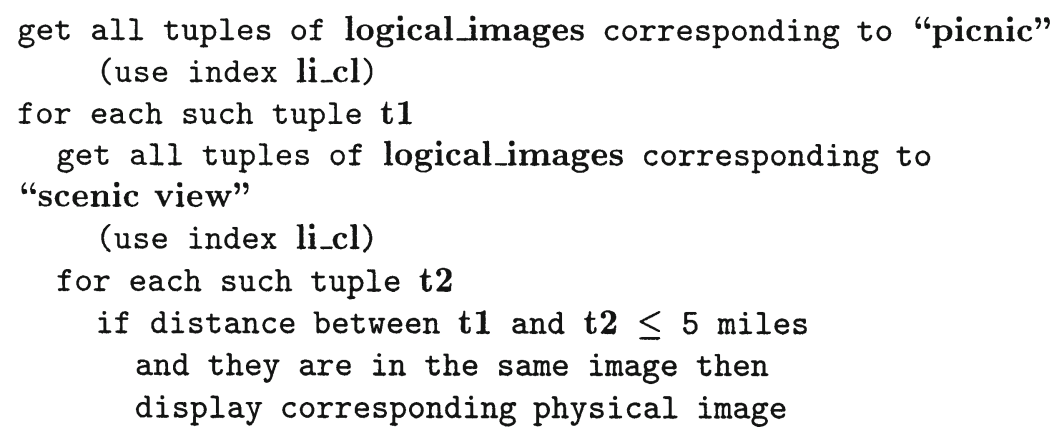

Plan $\mathbf{P 2 B}_{I}$ Search for "picnic" tuples using an alphanumeric index on class and search for "scenic view" tuples using a spatial index on loc.

get all tuples of logical_images corresponding to "picnic" (use index li_cl) 
(using the incremental nearest neighbor operation)

for each one of these points $p$

if $p$ is a "scenic view"' and in same image then

display the corresponding physical image

Plan P2A $\mathbf{A}_{P}$ Search both the picnic and scenic view partitions sequentially.

$$
\begin{aligned}
& \text { for each tuple t1 of the "picnic" partition } \\
& \text { for each tuple t2 of the "scenic view" partition } \\
& \text { if distance between t1.loc and t2.loc } \leq 5 \text { miles } \\
& \text { and they are in the same image then } \\
& \text { display the corresponding physical image }
\end{aligned}
$$

Plan P2B $_{P}$ Search the picnic partition sequentially, and search the scenic view partition using the spatial index on loc.

$$
\begin{aligned}
& \text { for each tuple t1 of the "picnic" partition } \\
& \text { get all points within } 5 \text { miles of t1.loc } \\
& \text { in the "scenic view" partition } \\
& \text { for each one of these points } p \\
& \text { if } p \text { is in the same image as t1 then } \\
& \text { display the corresponding physical image }
\end{aligned}
$$

\subsubsection{Cost Analysis}

\begin{tabular}{|l|l|}
\hline Name & Meaning \\
\hline$c_{r}$ & accessing a tuple by tid (random order) \\
$c_{s q}$ & accessing a tuple in sequential order \\
$c_{s q f}$ & accessing the first tuple of a relation \\
$c_{a f}$ & "find first" operation on an alphanumeric index \\
$c_{a n}$ & "find next" operation on an alphanumeric index \\
$c_{l s f}$ & "find nearest neighbor" operation on a location space index \\
$c_{l s n}$ & "find next nearest neighbor" operation on a location space index \\
$c_{f s f}$ & "find nearest neighbor" operation on a feature space index \\
$c_{f s n}$ & "find next nearest neighbor" operation on a feature space index \\
$c_{s c}$ & string comparison \\
$c_{l s d}$ & distance computation in location space \\
$c_{f s d}$ & weighted distance computation in feature space \\
\hline
\end{tabular}

Table 24.1: Costs of basic operations used in query processing.

In order to estimate the costs of each plan, we must make assumptions about the data distribution and the costs of the various operations. Table 24.1 contains a tabulation of the costs of basic operations used to process queries. The cost of many of these operations is a function of the relation on which they operate. $c_{x(y)}$ is the cost of performing operation $x$ on relation or index $y$. li 
stands for logical_images. The cost of accessing the physical_images relation to retrieve the result image and the cost of the "display" operation are not included as it is always the same regardless of the selected execution plan. Let $N_{p i c}$ and $N_{s v}$ be the number of tuples from class "picnic" and "scenic view", respectively. Let $B_{p i c}$ and $B_{s v}$ be the number of disk blocks containing tuples from class "picnic" and "scenic view", respectively.

Equations 24.1, and 24.2 estimate the cost of responding to query 1 using the integrated and partitioned organizations, respectively.

$$
\begin{aligned}
C_{1_{I}} & =c_{a f\left(l i i_{-} l\right)}+N_{s v} \times\left(c_{r(l i)}+c_{a n\left(l i_{-} c l\right)}\right) \\
C_{1_{P}} & =N_{s v} \times c_{s q\left(s v_{-} p a r t\right)}
\end{aligned}
$$

One difference between $C_{1_{I}}$ and $C_{1_{P}}$ is that in the integrated organization, there is an "alphanumeric find" operation on index li_cl that is not necessary in the partitioned organization. It is required in order to find the first scenic view tuple in this index. In addition, one more random access is required for each scenic view tuple in order to get the img_id from the logical_images relation. The other difference is that there are $N_{s v}$ alphanumeric next operations in the integrated organization compared with $N_{s v}$ sequential access operations in the partitioned organizations. The reason for this is that in the partitioned organization, the relation is scanned directly, whereas in the integrated organization, the index is scanned.

$$
\begin{aligned}
C_{2 A_{I}}= & c_{a f\left(l i \_c l\right)}+N_{p i c} \times\left(c_{r(l i)}+c_{a n\left(l i i_{-} l\right)}\right)+ \\
& B_{p i c} \times\left[c_{a f\left(l i_{-} l\right)}+N_{s v} \times\left(c_{r(l i)}+c_{a n\left(l i \_c l\right)}\right)\right]+ \\
& N_{p i c} \times N_{s v} \times c_{l s d} \\
C_{2 A_{P}}= & N_{p i c} \times c_{s q\left(p i_{-} p a r t\right)}+ \\
& B_{p i c} \times\left[c_{s q f\left(s v_{-} p a r t\right)}+N_{s v} \times c_{s q\left(s v_{-} \text {part }\right)}\right]+ \\
& N_{p i c} \times N_{s v} \times c_{l s d}
\end{aligned}
$$

Equations 24.3 and 24.4 estimate the cost of responding to query 2 with plan A using the integrated and partitioned organizations, respectively. In both equations, the first line is the cost of reading all pic tuples, the second line is the cost of reading all sv tuples for each block, and the last line is the cost of checking the distance between each (pic,sv) pair. $N_{I n C_{2}}$ denotes the average number of tuples in the circular range specified in query $2\left(C_{2}\right) . \quad N_{s v_{-} I n C_{2}}$ denotes the average number of scenic view tuples in $C_{2}$. Assuming a uniform distribution of symbols in space (i.e., there is an equal number of symbols in any given area), then $N_{I n C_{2}}=\frac{\operatorname{area}\left(C_{2}\right)}{A} \times N$, where $N$ is the total number of tuples in the logical_images relation, $A$ is the area covered by these tuples, and $C_{2}$ is the circular range specified in query 2. Assuming a uniform distribution of classifications among the symbols (i.e., there is an equal number of symbols from each classification in any group of symbols), then $N_{s v_{-} I n C_{2}}=\frac{\operatorname{area}\left(C_{2}\right)}{A} \times \frac{N}{C L}$, where $C L$ is the number of different classifications in the database. 
If these assumptions about the distribution of the classifications among symbols do not hold, then other methods are required to estimate the number of scenic view tuples in a given area. The portion of all tuples that belong to each classification can be recorded when populating the database by checking the class attribute and tallying the number for each classification. This data can then be used to estimate the distribution of the classifications among the symbols. Assuming that the distribution of classifications among any group of symbols is equal to the the total database distribution (i.e., the portion of tuples from each classification among any given group of symbols is equal to the portion of tuples from each classification in the entire database), then $N_{s v_{-} I n C_{2}}=\frac{\operatorname{area}\left(C_{2}\right)}{A} \times s v_{p} \times N$, where $s v_{p}$ is the portion of the database tuples that belong to the "scenic view" class.

Plan P2A $\mathrm{A}_{C}$ performs a spatial join operation on the results of two selection operations on relation logical_images. The first select operation extracts all tuples of the relation that are of class "picnic", while the second select operation extracts all tuples of the relation that are of class "scenic view". The results of these two select operations are then joined according to a predicate based on the loc attribute. In our implementation of plan P2A $\mathrm{A}_{C}$, we perform the select and join operations simultaneously using a block nested loop join algorithm as follows. One of the classes is designated as the inner class, and the other is designated as the outer class. One block of tuples belonging into the outer class are read into a memory-resident buffer (using the index on attribute class). All tuples of the inner class are then read (one block at a time using the index on attribute class) and spatially joined with all tuples of the outer class that are in memory (by computing the predicate on the spatial attribute). This process is repeated with the next block of tuples of the outer class, until all tuples of the outer class have been read.

The main difference between $C_{2 A_{I}}$ and $C_{2 A_{P}}$ is that in the integrated organization the index is scanned sequentially, whereas in the partitioned organization the relation corresponding to the scenic view partition is scanned sequentially (as in the case of query 1). As a result, once again, there are considerably more "random access" operations in the integrated organization than in the partitioned organization.

Equations 24.5 and 24.6 estimate the cost of responding to query 2 with plan B using the integrated and partitioned organizations, respectively. Again, as in equations 24.3 and 24.4, the first line is the cost of reading all pic tuples, but the second line is the cost of finding sv tuples in the range (using index li_loc) for each pic.

$$
\begin{aligned}
C_{2 B_{I}}= & c_{a f\left(l i \_c l\right)}+N_{p i c} \times\left[c_{r(l i)}+c_{a n\left(l i \_c l\right)}\right]+ \\
& N_{p i c} \times\left[c_{l s f\left(l i \_l o c\right)}+N_{I n C_{2}} \times\left(c_{r(l i)}+c_{s c}+c_{l s n\left(l i \_l o c\right)}\right)\right] \\
C_{2 B_{P}}= & N_{p i c} \times c_{s q\left(p i_{-} p a r t\right)}+ \\
& N_{p i c} \times c_{l s f\left(s v \_l o c\right)}+N_{s v_{-} I n C_{2}} \times\left(c_{r\left(s v \_p a r t\right)}+c_{l s n\left(s v \_l o c\right)}\right)
\end{aligned}
$$

The main difference between $C_{2 B_{I}}$ and $C_{2 B_{P}}$ is in the number of locationspace "find next" operations and the number of random access operations. 
In the integrated organization, all tuples $\mathrm{t}$ of any class in circle $C_{2}$ are retrieved from the spatial index. The class of $t$ is then retrieved from the logical_images relation to see if it corresponds to a "scenic view". This requires a random access operation for each tuple in $C_{2}$. On the other hand, in the partitioned organization, only tuples of type "scenic view" are retrieved by the spatial index. Thus, there is no need for an additional random access to check the class of the tuple. In addition, since only a subset of the tuples in circle $C_{2}$ are "scenic view" tuples, the number of items retrieved by the spatial query in the integrated organization (i.e., $N_{I n C_{2}}$ ) is larger than the number of items retrieved by the spatial query in the partitioned organization (i.e., $N_{s v_{-} I n C_{2}}$ ).

Another significant difference between $C_{2 B_{I}}$ and $C_{2 B_{P}}$ is that the spatial index on which the search is performed is smaller in the partitioned organization since it only contains "scenic view" tuples (i.e., $\mid$ sv_loc $|<| 1$ i_loc $\mid$ ). As a result, $c_{l s f\left(s v_{-} l o c\right)}$ and $c_{l s n\left(s v \_l o c\right)}$ are less than $c_{l s f\left(l i \_l o c\right)}$ and $c_{l s n\left(l i \_l o c\right)}$, respectively. Therefore, the difference between the total cost of plan P2B in the partitioned organization and the total cost of plan P2B in the integrated organization is greater than in the case of plan P2A. The plan for the partitioned organization can be further improved by implementing a more sophisticated form of the spatial join operation between the two relations scenic_part and pi-part which correspond to "scenic view" and "picnic", respectively. The overall idea is that the join can be computed more efficiently by traversing both indices in parallel in such a way as to avoid comparing tuples which cannot satisfy the join condition. This operation has not been implemented in SAND yet. Once it is added, plan $P 2 B_{P}$ will be revised accordingly.

It is interesting to compare the costs of answering query 2 for one particular organization using plans $\mathrm{P} 2 \mathrm{~A}$ and $\mathrm{P} 2 \mathrm{~B}$. For the integrated organization, we compare equations 24.3 and 24.5. In plan $\mathrm{P}^{2} \mathrm{~A}_{I}$, both relations are scanned sequentially via the alphanumeric index 1i_cl. For each picnic tuple, each scenic view tuple is checked to determine whether or not it is within the specified range. Thus, the total number of distance computations is $N_{p i c} \times N_{s v}$. In addition, the same number of random access operations are also required in order to get the locations from the logical_images relations. In plan $\mathrm{P}^{2} \mathrm{~B}_{I}$, the spatial index is used and thus only tuples that are within the specified range need to be examined. The cost of this is the overhead involved in using the spatial index. In this case, this cost is $N_{\text {pic }}$ location-space "find first" operations, and $N_{p i c} \times N_{I n C_{2}}$ location-space "find next" operations. These spatial operations involve distance computations as part of the incremental nearest neighbor operation. However, there is no need for any distance computations as part of the plan itself. Whether plan $\mathrm{P}^{2} \mathrm{~A}_{I}$ or plan $\mathrm{P}^{2} \mathrm{~B}_{I}$ is better depends on the size of the data set, the portion of these tuples that belong to each classification (termed the contextual selectivity), and on the portion of all tuples that fall in the range specified by the spatial component (termed the spatial selectivity). Assuming a high spatial selectivity (i.e., that the number of tuples in the spatial range is much smaller than the total number of tuples in the 
data set), plan $\mathrm{P}^{2} \mathrm{~B}_{I}$ should prove to be much more efficient than plan $\mathrm{P}^{2} \mathrm{~A}_{I}$. However, if the spatial selectivity is low, then plan $\mathrm{P}_{2} \mathrm{~A}_{I}$ may prove to be better. Similar observations can be made about the partitioned organization by comparing equations 24.4 and 24.6. Once a more efficient spatial join operator is implemented, as mentioned above, the difference will be even greater.

\subsection{CONCLUDING REMARKS}

Two different data organizations (integrated and partitioned) for storing logical images in relational tables were proposed. They differ in the way that the logical images are stored. Sample queries and execution plans to answer these queries were described for both organizations. Analytical cost analyses of these execution plans were given that indicated that the partitioned data organization is more efficient for queries that consist of both contextual and spatial specifications. On the other hand, the integrated organization is better for purely spatial specifications. Both organizations gave similar results for queries that consist of purely contextual specification.

Our definition of the class of images that we can handle is rather strict. Some of these restrictions can be relaxed. In particular, the requirement that there exists a function $f$ which when given a symbol $s$ and a class $C$ returns a value between 0 and 1 indicating the certainty that $s$ belongs to $C$ can be omitted. In this case, we can store the feature vectors in the database rather than the classifications. For a comparison of using these two approaches, see [16]. Of course, more elaborate indexing methods are then required to respond to queries such as those presented in this paper.

\subsection{ACKNOWLEDGMENTS}

This work was supported in part by the National Science Foundation under Grants CDA-950-3994 and IRI-97-12715. We are grateful to Karttakeskus, Map Center, Helsinki, Finland for providing us the map data.

\section{References}

[1] W. G. Aref and H. Samet. Optimization strategies for spatial query processing. In Proc. of the 17th Intl. Conf. on Very Large Data Bases, pp. 81-90, Barcelona, Sept. 1991.

[2] S. K. Chang, E. Jungert, and Y. Li. The design of pictorial databases based upon the theory of symbolic projections. In Design and Implementation of Large Spatial Databases - 1st Symp., SSD'89, pp. 303-323, Santa Barbara, CA, July 1989. (Also Springer-Verlag Lecture Notes in Computer Science 409).

[3] C. Esperança and H. Samet. Spatial database programming using SAND. In Proc. of the 7th Intl. Symp. on Spatial Data Handling, vol. 2, pp. A29A42, Delft, The Netherlands, Aug. 1996. 
[4] C. Faloutsos. Access methods for text. ACM Comp. Surveys, 17(1):49-74, Mar. 1985.

[5] V. Gudivada and V. Raghavan. Design and evaluation of algorithms for image retrieval by spatial similarity. ACM Trans. Info. Syst., 13(2):115144, Apr. 1995.

[6] A. Gupta, T. Weymouth, and R. Jain. Semantic queries with pictures: the VIMSYS model. In Proc. of the 17th Intl. Conf. on Very Large Databases, pp. 69-79, Barcelona, Spain, Sept. 1991.

[7] G. R. Hjaltason and H. Samet. Ranking in spatial databases. In Advances in Spatial Databases - 4th Intl. Symp., SSD'95, pp. 83-95, Portland, ME, Aug. 1995. (Also Springer-Verlag Lecture Notes in Computer Science 951).

[8] R. Jain. NSF workshop on visual information management systems. SIGMOD RECORD, 22(3):57-75, Sept. 1993.

[9] R. Kasturi, R. Raman, and C. Chennubhotla. Document image analysis an overview of techniques for graphics recognition. In Proc. of the IAPR Workshop on Syntactic and Structural Pat. Rec., pp. 192-230, Murray Hill, NJ, June 1990.

[10] R. C. Nelson and H. Samet. A consistent hierarchical representation for vector data. Computer Graphics, 20(4):197-206, Aug. 1986. (Also Proc. of SIGGRAPH'86, Dallas, Aug. 1986).

[11] W. Niblack, R. Barber, W. Equitz, M. Flickner, E. Glasman, D. Petkovic, and P. Yanker. The QBIC project: Querying images by content using color, texture, and shape. In Proc. of the SPIE, Storage and Retrieval of Image and Video Databases, vol. 1908, pp. 173-187, San Jose, CA, Feb. 1993.

[12] V. Oria, B. Xu, and M. T. Tamer. VisualMOQL: A visual query language for image databases. In Proc. of the IFIP 2.6 4th Working Conf. on Visual Database Systems (VDB-4), pp. 186-191, L'Aquila, Italy, May 1998.

[13] A. Pentland, R. W. Picard, and S. Sclaroff. Photobook: Content-based manipulation of image databases. In Proc. of the SPIE, Storage and Retrieval of Image and Video Databases II, vol. 2185, pp. 34-47, San Jose, CA, Feb. 1994.

[14] D. Rotem. Spatial join indices. In Proc. of the 7th Intl. Conf. on Data Eng., pp. 500-509, Kobe, Japan, April 1991. IEEE Computer Society Press.

[15] H. Samet and A. Soffer. MAGELLAN: Map acquisition of geographic labels by legend analysis. Intl. Journal of Document Analysis and Recognition, 1(2):89-101, June 1998.

[16] A. Soffer and H. Samet. Two approaches for integrating symbolic images into a multimedia database system: a comparative study. VLDB Journal, to appear.

[17] M. Swain. Interactive indexing into image databases. In Proc. of the SPIE, Storage and Retrieval for Image and Video Databases, vol. 1908, pp. 95-103, San Jose, CA, Feb. 1993. 
[18] Virage. Virage web site. http://www.virage.com. 